\title{
TWO VERSIONS OF NAKAYAMA LEMMA FOR MULTIPLICATION MODULES
}

\author{
REZA AMERI
}

Received 24 November 2003

\begin{abstract}
The aim of this note is to generalize the Nakayama lemma to a class of multiplication modules over commutative rings with identity. In this note, by considering the notion of multiplication modules and the product of submodules of them, we state and prove two versions of Nakayama lemma for such modules. In the first version we give some equivalent conditions for faithful finitely generated multiplication modules, and in the second version we give them for faithful multiplication modules with a minimal generating set.
\end{abstract}

2000 Mathematics Subject Classification: 16D10.

1. Introduction. Let $R$ be a commutative ring with identity and let $M$ be a unitary $R$-module. Then $M$ is called a multiplication $R$-module provided for each submodule $N$ of $M$ there exists an ideal $I$ of $R$ such that $N=I M$, we call $I$ a presentation ideal of $N$. Note that our definitions agree with [2, 3], but in [5], the term multiplication module is used in a different way. (In this note, an $R$-module $M$ is multiplication if and only if every submodule of $M$ is a multiplication module in the above sense.)

If $N$ and $K$ are multiplication submodules of $M$, then $N K$, the product of $N$ and $K$, is defined as $I J M$, where $I$ and $J$ are presentation ideals of $N$ and $K$, respectively [1]. For a module $M$, the radical of $M$, denoted by $\operatorname{rad}(M)$, is the intersection of all maximal submodules of $M$ if they exist, and $M$ otherwise (see [3, 4]). We denote the Jacobson radical of the ring $R$, the intersection of all maximal ideals of $R$, by $J(R)$. Recently, multiplication modules have been studied in a number of papers, see, for example, $[1,2,3,5]$. The author in [1] states and proves a version of Nakayama lemma for multiplication modules (see [1, Theorem 3.23]). But the proof of (ii) $\Rightarrow$ (iii) of this theorem needs to be amended. In this note, first we modify the proof of this theorem, and then we obtain another version of Nakayama lemma for multiplication modules by replacing the finitely generated condition with a minimal generating set for them.

Throughout this note, $R$ denotes a commutative ring with identity and all related modules are unitary $R$-modules. All definitions and notations follow from $[1,2,3]$.

\section{Main results}

LEMMA 2.1. Let $M$ be a finitely generated $R$-module and $M=I M$ for some ideal $I$ of $R$. Then $(1-r) M=0$ for some $r \in R$. 
Proof. Let $M$ be generated by elements $x_{1}, x_{2}, \ldots, x_{n}$. Since $M=I M$, every $x_{i}$ can be written as an $I$-linear combination of $x_{1}, x_{2}, \ldots, x_{n}$. Thus we have

$$
x_{i}=a_{i 1} x_{1}+a_{i 2} x_{2}+\cdots+a_{i i} x_{i}+\cdots+a_{i n} x_{n}, \quad 1 \leq i \leq n, a_{i 1}, \ldots, a_{i n} \in I .
$$

Then

$$
\begin{aligned}
\left(1-a_{i i}\right) x_{i}= & a_{i 1} x_{1}+a_{i 2} x_{2}+\cdots+a_{i i-1} x_{i-1} \\
& +a_{i i+1} x_{i+1}+\cdots+a_{i n} x_{n}, \quad 1 \leq i \leq n, a_{i 1}, \ldots, a_{i n} \in I .
\end{aligned}
$$

Recursively from (2.2) we can find each $x_{i}$ of the form $\left(1-r_{i}\right) x_{i}=0$ for some $r_{i} \in R$, $1 \leq i \leq n$. Then we have $\left(1-r_{1}\right)\left(1-r_{2}\right) \cdots\left(1-r_{n}\right)=1-r$ for some $r \in I$. Thus $(1-$ $r) x_{i}=0$, for all $i, 1 \leq i \leq n$. This completes the proof.

LEMMA 2.2. If $M$ is a faithful multiplication $R$-module, then $\operatorname{rad}(M)=J(R) M$.

Proof. Let $\mathcal{M}$ denote the collection of all maximal ideals of $R$. Set $P_{2}(M)=\{P \in \mathcal{M} \mid$ $\operatorname{ann}(M) \subseteq P\}$. Since $M$ is faithful, $P_{2}(M)=M$. Then, by [1, Theorem 2.13], $\operatorname{rad}(M)=$ $\left(\bigcap_{P \in P_{2}(M)} P\right) M=J(R) M$.

Definition 2.3 (See [5]). Let $M$ be an $R$-module. An element $u \in M$ is called a unit element if $\langle u\rangle=M$.

In the next result we modify the proof of [1, Theorem 3.23].

THEOREM 2.4 (first version of Nakayama lemma). Let $M$ be a faithful multiplication $R$-module. Then, for every submodule $N$ of $M$, the following conditions are equivalent:

(i) $N$ is contained in $\operatorname{rad}(M)$,

(ii) if $u$ is a unit in $M$, then $u-r x$ is a unit for all $r \in R$ and for all $x \in N$,

(iii) if $M$ is a finitely generated $R$-module such that $N M=M$, then $M=0$,

(iv) if $M$ is finitely generated and $K, N$ are submodules of $M$ such that $M=N M+K$, then $M=K$.

Proof. (i) $\Rightarrow$ (ii). The proof is the same as in the proof of [1, Theorem 3.23].

(i) $\Rightarrow$ (ii). Since $u$ is a unit in $M$, then $M=\langle u\rangle$ by [1, Theorem 3.19]. By contradiction let $N \nsubseteq \operatorname{rad}(M)$. Then there exists a maximal submodule $K$ of $M$ such that $N \nsubseteq K$. Thus, there exists $x \in N \backslash K$, and hence $\langle x\rangle+K=M$ by the maximality of $K$. Thus, $u=r x+a$ for some $r \in R$ and $a \in K$. Then, by hypothesis, $a=u-r x$ is a unit, and hence $K=M$, which is a contradiction. Therefore, $N \subseteq \operatorname{rad}(M)$.

(i) $\Rightarrow$ (iii). Let $I$ be a presentation ideal of $N$, that is, $N=I M$. Then, by the product of two submodules (see [1, Definition 3.3]) and the hypothesis, we have $M=N M=I M \cdot R M=$ $I M$. On the other hand, by hypothesis, we have $N \subseteq \operatorname{rad}(M)$, and from Lemma 2.2 we conclude that $M=J(R) M$. Thus, by Lemma 2.1, there exists an $r \in J(R)$ such that $(1-r) M=0$, and hence $M=0$ since $1-r$ is a unit in $R$.

(iii) $\Rightarrow$ (iv). By [1, Corollary 3.22], $K / N$ is a multiplication $R$-module. Now, it is easy to verify that $(K+N) / K(M / K)=M / K$, and hence, by (iii), we must have $M / K=0$, and hence $M=K$.

(iv) $\Rightarrow$ (i). Let $K$ be any maximal submodule of $M$; then $K \subseteq N M+K$. Consequently, $N M+M=M$ or $N M+M=K$ by the maximality of $K$. If $N M+K=M$, then, by (iv), 
we must have $K=M$, a contradiction. Thus, $K=N M+K$, and hence $N \subseteq N M \subseteq K$. Therefore, $N$ is contained in every maximal submodule of $M$.

Definition 2.5. Let $M$ be an $R$-module. A subset $X$ of $M$ is called a minimal generating set if $\langle X\rangle=M$ and no proper subset of $X$ generates $M$.

THEOREM 2.6 (second version of Nakayama lemma). Let $M$ be a faithful multiplication $R$-module with a minimal generating set. Then, for every submodule $N$ of $M$, the following conditions are equivalent:

(i) $N$ is contained in $\operatorname{rad}(M)$,

(ii) if $N M=M$, then $M=0$,

(iii) if $K$ is a submodule of $M$ such that $M=N M+K$, then $M=K$.

Proof. (i) $\Rightarrow$ (ii). Let $X$ be a minimal generating set of $M$. If $M \neq 0$, then consider $m_{1}, m_{1} \neq 0$, by the minimality of $X$. Now, let $I$ be a presentation ideal of $N$. Then $N M=M$ implies that $N=I M \cdot M=M$, and since $M$ is faithful, by Lemma 2.2, we have $N \subseteq \operatorname{rad}(M)=J(R) M$, and hence $M=J(R) M$. Thus, $m_{1}=j_{1} m_{1}+j_{2} m_{2}+\cdots+j_{n} m_{n}$, $j_{i} \in J(R), m_{i} \in X$, whence $j_{1} m_{1}=m_{1}$. If $n=1$, then $\left(1-j_{1}\right) m_{1}=0$; since $1-j_{1}$ is a unit in $R, m_{1}=0$, and for $n>1$,

$$
\left(1-j_{1}\right) m_{1}=j_{2} m_{2}+\cdots+j_{n} m_{n}
$$

Since $1-j_{1}$ is a unit in $R, m_{1}=\left(1-j_{1}\right)^{-1} j_{2} m_{2}+\cdots+\left(1-j_{1}\right)^{-1} j_{n} m_{n}$. Thus, for $n>1$, $m_{1}$ is a linear combination of $m_{2}, m_{3}, \ldots, m_{n}$. Consequently, $\left\{m_{2}, \ldots, m_{n}\right\}$ generates $M$, which contradicts the choice of $X$.

The proofs of (ii) $\Rightarrow$ (iii) and (iii) $\Rightarrow$ (i) are the same as the proofs of (iii) $\Rightarrow$ (iv) and (iv) $\Rightarrow$ (i) of Theorem 2.4.

ACKNOWLEDGMENTS. The author is thankful to the referees for their comments and suggestions. This work was carried out during a sabbatical leave of the author in the University of Montreal. The author would like to thank the Department of Mathematics and Statistics of University of Montreal for hospitality and the University of Mazandaran for financial support.

\section{REFERENCES}

[1] R. Ameri, On the prime submodules of multiplication modules, Int. J. Math. Math. Sci. 2003 (2003), no. 27, 1715-1724.

[2] A. Barnard, Multiplication modules, J. Algebra 71 (1981), no. 1, 174-178.

[3] Z. A. El-Bast and P. F. Smith, Multiplication modules, Comm. Algebra 16 (1988), no. 4, 755779.

[4] R. L. McCasland and M. E. Moore, On radicals of submodules of finitely generated modules, Canad. Math. Bull. 29 (1986), no. 1, 37-39.

[5] S. Singh and F. Mehdi, Multiplication modules, Canad. Math. Bull. 21 (1969), 1057-1061.

Reza Ameri: Department of Mathematics and Statistics, Faculty of Basic Sciences, University of Mazandaran, Babolsar 47415, Iran

E-mail address: ameri@umz.ac.ir 


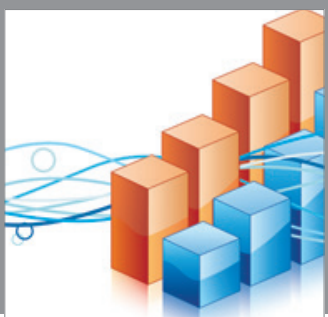

Advances in

Operations Research

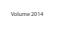

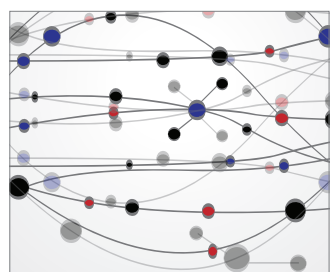

\section{The Scientific} World Journal
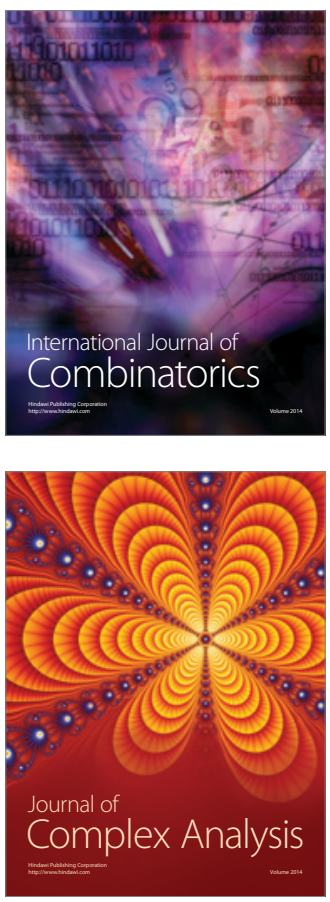

International Journal of

Mathematics and

Mathematical

Sciences
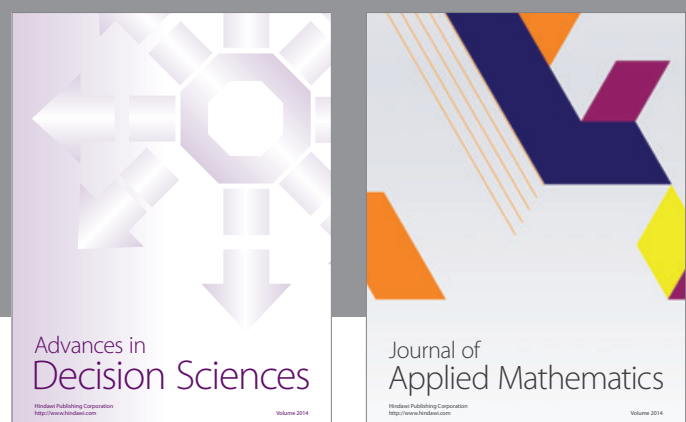

Journal of

Applied Mathematics
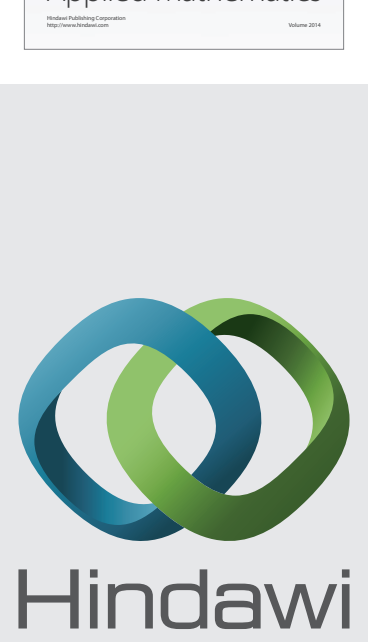

Submit your manuscripts at http://www.hindawi.com
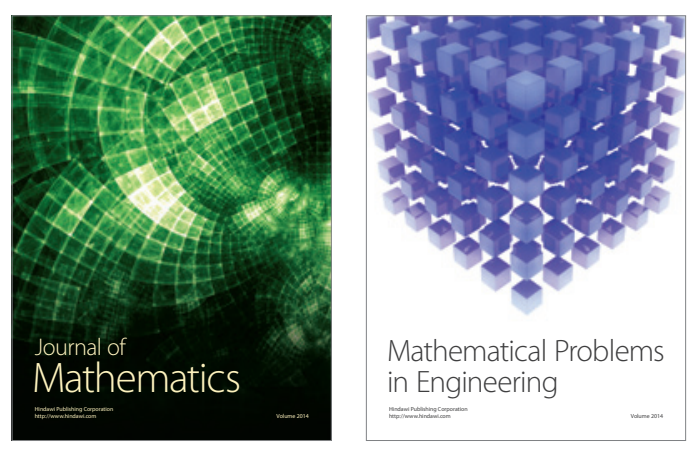

Mathematical Problems in Engineering
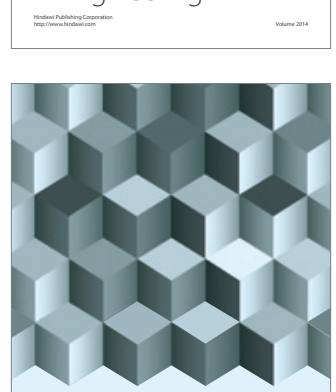

Journal of

Function Spaces
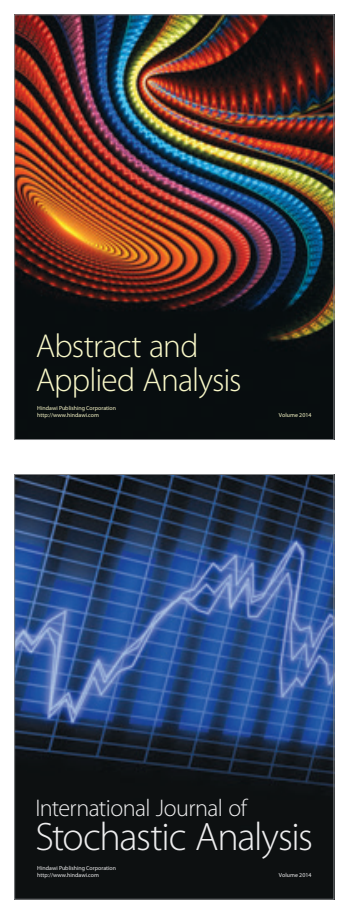

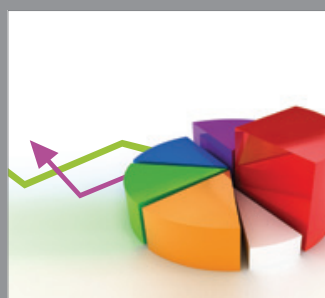

ournal of

Probability and Statistics

Promensencen
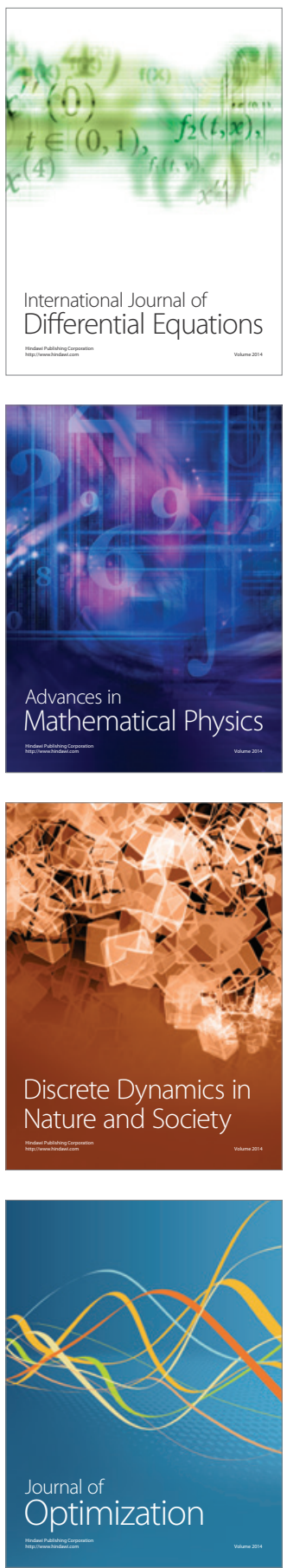